\title{
NOTE
}

\section{THE REACTION OF CYSTEINE AND RELATED COMPOUNDS WITH PENICILLINS AND CEPHALOSPORINS*}

\author{
Eugene S. Wagner** and Marvin Gorman \\ Lilly Research Laboratories, \\ Eli Lilly and Company, \\ Indianapolis, Indiana, U.S.A.
}

(Received for publication May 17, 1971)

Evidence has been published for the reaction of penicillenic acid ${ }^{1,2)}$ and for the direct interaction of penicillin ${ }^{3,4,5)}$ with free amino groups of protein to form penicilloyl compounds. These penicilloyl derivatives are reported to be the major determinant in penicillin allergy ${ }^{4,6)}$.

Our initial observation that benzylpenicillenic acid, one of the major degradation products of benzylpenicillin, is converted by ethanethiol to $\alpha$-ethylthiobenzylpenicilloate ${ }^{7)}$ generated an interest in the action of thiolcontaining compounds on the $\beta$-lactam of penicillins. The possibility that benzylpenicillenic acid or benzylpenicillin, by reaction with the sulfhydryl groups of protein, could form a determinant responsible for an allergic response is of theoretical interest.

A comparison of the reactivity of benzylpenicillin and of benzylpenicillenic acid with thiol-containing nucleophiles was undertaken. The progress of the reactions was monitored spectrophotometrically. Within minutes the reaction of benzylpenicillenic acid with compounds containing free sulfhydryl groups is complete (Table 1). The results indicate that a large variety of compounds react, and only the absence of a free sulfhydryl interferes with reactivity. As reported earlier ${ }^{8)}$ the reaction of benzylpenicillin with 2-mercaptoamines having both a sulfhydryl group and an amino group also is complete within minutes. When either the sulfhydryl or the amino group of the 2-mercaptoamine is absent or blocked, no reaction is observed with benzylpenicillin.

Since the ability to form penicilloylthioesters differs with benzylpenicillenic acid and benzylpenicillin, we investigated the reactivity of several cephalosporin antibiotics with 2-mercaptoamines. The stabilities of a variety of penicillins and cephalosporins in the presence of cysteine were measured (Table 2). At pH 7.5 and room temperature, both 3-methyl cephem and 3-acetoxymethyl cephem derivatives are unreacted after four

Table 1. Reaction of benzylpenicillenic acid and benzylpenicillin with thiol-containing compounds

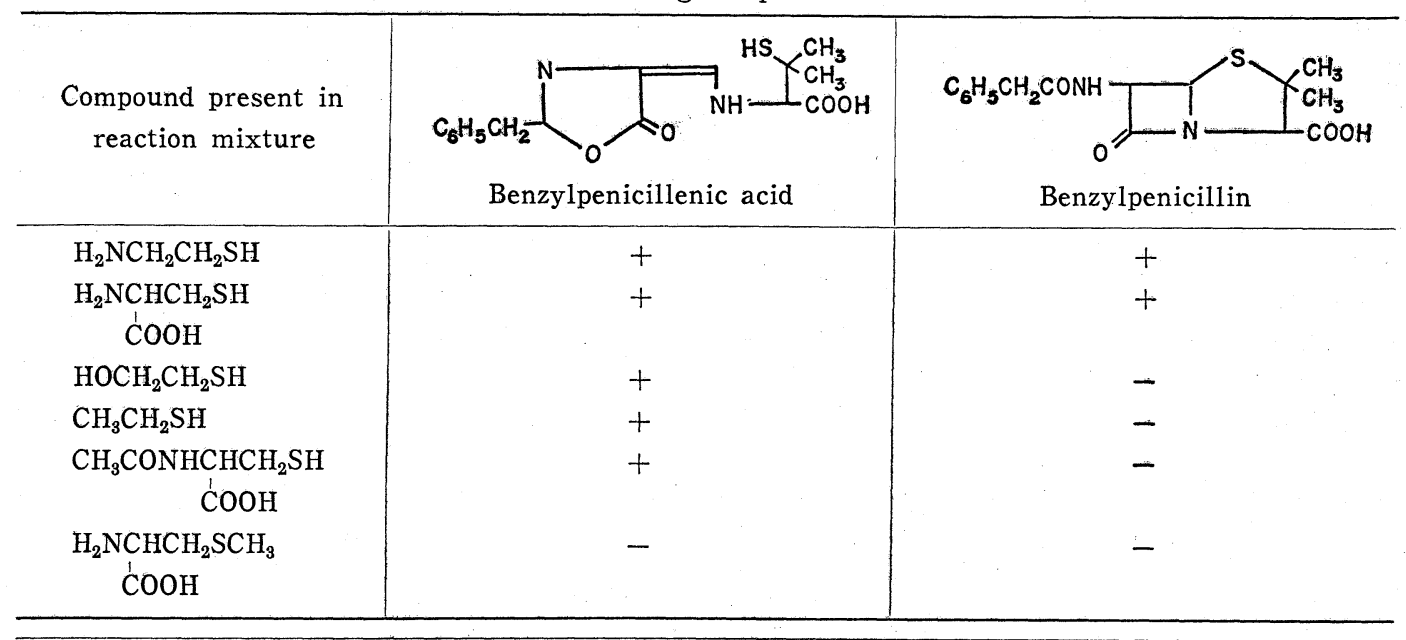

* Presented in part at the VIII th Interscience Conference on Antimicrobial Agents and Chemotherapy, New York, New York, October 1968.

** Present address : Ball State University, Department of Chemistry, Muncie, Indiana, 47304, U.S.A. 
Table 2. Reaction of penicillins and cephalosporins with"cysteine

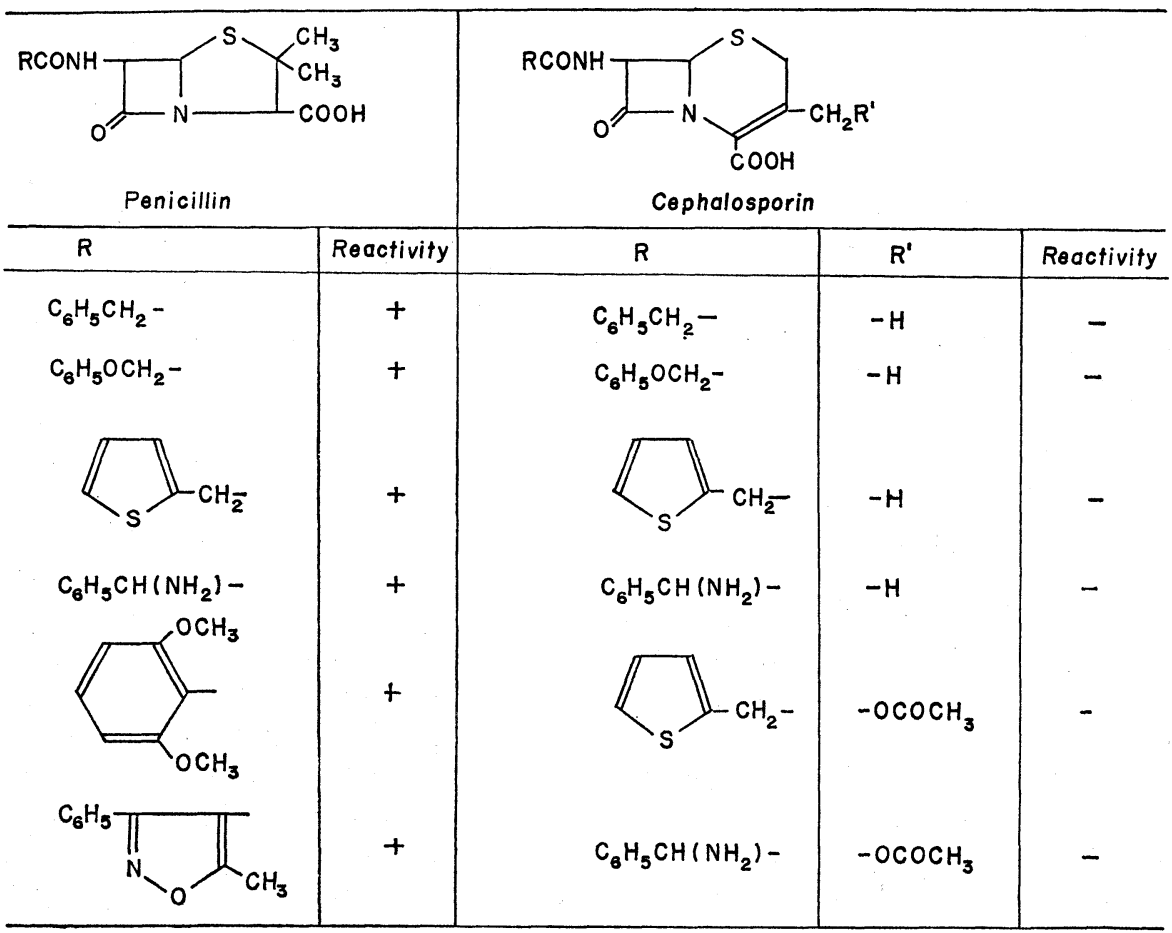

hours, while under identical conditions the corresponding penicillins react completely within one hour.

In order to establish whether this difference in reactivity was related to the presence of a side chain on the antibiotics, 6-aminopenicillanic acid (6-APA) and 7aminocephalosporanic acid (7-ACA) were treated with cysteine. After four hours the $\beta$-lactam band in the IR spectrum of the reaction mixture containing $6-\mathrm{APA}$ and cysteine disappears. Under identical conditions there is no effect on the $\beta$-lactam of 7-ACA, since the control 7-ACA (lacking cysteine) shows an identical $\beta$-lactam (1800 $\left.\mathrm{cm}^{-1}\right)$ to ester $\left(1740 \mathrm{~cm}^{-1}\right)$ ratio as the reaction mixture.

The selectivity of this reaction indicates that penicillin $\beta$-lactam activity may be removed in the presence of cephalosporins. The results of the following experiment verified this contention. Cephalothin, 7(2-thienylacetamido)cephalosporanic acid, was contaminated to the extent of $1 \%$ with phenoxymethylpenicillin. Samples were taken from a mixture of this cephalosporin, phenoxymethylpenicillin, and cysteine at $\mathrm{pH}$ 7.5 initially and after one and two hours. A microbiological assay of the one hour sample showed a trace amount of the original penicillin activity and after two hours the activity due to penicillin is gone. Mixtures of the same cephalosporin and phenoxymethylpenicillin without cysteine in $\mathrm{pH} 7.5$ buffer and the same cephalosporin in buffer served as controls. Duplicates of all samples assayed were treated with penicillinase to illustrate the disappearance of penicillin activity by a known agent.

We have established that the reactivity of penicillenic acids, penicillins, and cephalosporins with 2 -mercaptoamines differs. As a result, trace amounts of penicillin activity and, consequently, potential penicillin allergens can be destroyed in the presence of cephalosporins when more drastic physical treatment to remove possible contaminants would adversely effect cephalosporin activity. Studies are now in progress to determine whether structurally-modified cephalosporins interact with 2-mercaptoamines. 


\section{Experimental}

1. Phosphate Buffer Solution: $\mathrm{KH}_{2} \mathrm{PO}_{4}$ and $\mathrm{Na}_{2} \mathrm{HPO}_{4}$ (Mallinckrodt reagent) were used in the proper proportions for a $0.1 \mathrm{M}$ pH 7.5 aqueous solution.

2. All measurements of the presence of benzylpenicillenic acid were made in aqueous buffer on a Beckman Model DB spectrophotometer using $1.00 \mathrm{~cm}$ quartz cells. Spectrophotometric determination of the disappearance of benzylpenicillenic acid was followed at $322 \mathrm{~m} \mu$.

3. All measurements of the presence of the $\beta$-lactam of penicillins and cephalosporins were made on mineral oil mulls of the lyophilized residue of the reaction mixtures on a Beckman Model IR 12 Double Beam Recording Infrared Spectrometer.

4. 2-Mercaptoamine Compounds : 2Aminoethanethiol, cysteine, 2-mercaptoethanol, ethanethiol, $\mathrm{N}$-acetyl-L-cysteine, and S-methylcysteine were available commercially.

5. Penicillins and Cephalosporins: 6Aminopenicillanic acid, 7-aminocephalosporanic acid, benzylpenicillin, phenoxymethylpenicillin, 2-thienylmethylpenicillin, $\alpha-$ aminobenzylpenicillin, 2,6-dimethoxyphenylpenicillin, 4-(5-methyl-3-phenyl)isoxazolylpenicillin, 7-(2-phenylacetamido)-3-methyl3-cephem-4-carboxylic acid, 7-(2-phenoxyacetamido)-3-methyl-3-cephem-4-carboxylic acid, 7-(2-thienylacetamido)-3-methyl-3cephem-4-carboxylic acid, 7-(2-amino-2phenylacetamido)-3-methyl-3-cephem-4-carboxylic acid, 7-(2-thienylacetamido)cephalosporanic acid, and 7-(2-amino-2-phenylacetamido)cephalosporanic acid were obtained commercially when possible or were synthesized in the Lilly Research Laboratories.

6. Reaction of Benzylpenicillenic Acid with 2-Mercaptoamines: The initial reaction mixture of $3.0 \mathrm{ml}$ of $0.1 \mathrm{M}$ phosphate buffer at $\mathrm{pH} 7.5$ containing $1.0 \times 10^{-2} \mathrm{M}$ of the appropriate 2 -mercaptoamine was placed in a stoppered quartz $1.00 \mathrm{~cm}$ Beckman cell. A solution of benzylpenicillenic acid in absolute ethanol $(0.01 \mathrm{ml})$ was injected into the reaction cell, producing a concentration of $3.0 \times 10^{-5} \mathrm{M}$. Optical density measurements were then recorded as a function of time.

7. Reaction of Penicillins and Cephalosporins with Cysteine: A reaction mixture of $50 \mathrm{ml}$ of $0.1 \mathrm{M}$ phosphate buffer at $\mathrm{pH}$ 7.5 containing $300 \mathrm{mg}$ cysteine was added to a vessel containing $100 \mathrm{mg}$ of the appropriate penicillin or cephalosporin. The resulting mixture was stirred at room temperature for four hours and subsequently frozen and lyophilized. Controls were run for each antibiotic tested which excluded the cysteine. The resulting residues were submitted for IR analysis of the presence of $\beta$-lactam at $1760 \sim 1810 \mathrm{~cm}^{-1}$.

8. Reaction of 6-Aminopenicillanic Acid (6-APA) and 7-Aminocephalosporanic Acid (7-ACA) with Cysteine: Two reaction mixtures of $200 \mathrm{ml}$ of $0.1 \mathrm{M}$ phosphate buffer at $\mathrm{pH} 7.5$ containing $3.6 \mathrm{~g}$ cysteine were added to two vessels containing $1.0 \mathrm{~g} 6$ APA and $1.0 \mathrm{~g}$ 7-ACA, respectively. A third reaction mixture of $200 \mathrm{ml}$ of $0.1 \mathrm{M}$ phosphate buffer at $\mathrm{pH} 7.5$ containing $1.0 \mathrm{~g}$ 7-ACA served as a control. All three reaction mixtures were stirred at room temperature for four hours and subsequently frozen and lyophilized. The resulting residues were submitted for IR analysis of the presence of $\beta$-lactam at $1760 \sim 1810 \mathrm{~cm}^{-1}$ and ester at $1740 \mathrm{~cm}^{-1}$ in the case of $7-\mathrm{ACA}$.

9. Reaction of Phenoxymethylpenicillin with Cysteine in the Presence of a Cephalosporin. Isolation and Analysis of Penicillin : Three samples (labelled A, B, and C) were prepared; each contained $100 \mathrm{ml}$ of $0.1 \mathrm{M}$ phosphate buffer. Sample A contained $1.0 \mathrm{~g}$ of 7-(2-thienylacetamido)cephalosporanic acid, $10 \mathrm{mg}$ of phenoxymethylpenicillin, and $400 \mathrm{mg}$ cysteine; sample B contained $1.0 \mathrm{~g}$ of the same cephalosporin and $10 \mathrm{mg}$ of phenoxymethylpenicillin; and sample $\mathrm{C}$ contained the cephalosporin in buffer and served as a control. Samples were taken from samples $\mathrm{A}, \mathrm{B}$, and $\mathrm{C}$ initially and after one and two hours and added to $\mathrm{pH} 3.0$ water washed diisopropyl ether (DIPE). The $\mathrm{pH}$ of the aqueous phase was adjusted to 1.5 with $20 \% \quad \mathrm{H}_{2} \mathrm{SO}_{4}$ solution. The resulting slurry was filtered on $7 \mathrm{~cm}$ Whatman No. 1 filter paper on a Buchner funnel. The filtrate was placed in a separatory funnel 
and $100 \mathrm{ml}$ of the DIPE layer was applied to $1 / 4^{\prime \prime} \times 20^{\prime \prime}$ sheets of Whatman No. 4 paper which had been previously buffered with $0.5 \mathrm{M}$ to $0.75 \mathrm{M} \mathrm{pH} 6.2$ phosphate buffer. The chromatogram was allowed to equilibrate in a solvent chamber for approximately two hours before adding water saturated diethyl ether to the solvent tray. The chromatogram was allowed to develop down flow for approximately three hours followed by development on Sarcina lutea (Agar $\mathrm{pH}$ was 6.7 prior to pouring and plates were allowed to stand overnight in refrigerator prior to use). The appropriate standards were used for comparison of active material and duplicate samples were treated with penicillinase to illustrate the disappearance of penicillin activity by a known agent. The limit of detection was $0.5 \mathrm{ppm}$.

\section{Acknowledgement}

The authors are indebted to Mr. W. R. Brown for his excellent technical assistance and to $\mathrm{Mr}$. C. D. UNDERBRINK for the infrared analysis.

\section{References}

1) Levine, B. B. \& Z. Ovary: Studies on the mechanism of the formation of the penicillin antigen. J. Exp. Med. 114 : 875 904, 1961
2) De Weck, A. L. : Studies on penicillin hypersensitivity. Intern. Arch. Allergy Appl. Immunol. $21: 20 \sim 50.1962$

3) PARker, C. W. \& J. A. Thiel: Studies in human penicillin allergy: A comparison of various penicilloyl-polylysines. J. Lab. Clin. Med. 62 : 482 491, 1963

4) Batchelor, F. R.; J. M. Dewdney \& D. Gazzard: Penicillin allergy: The formation of the penicilloyl determinant. Nature $206: 362 \sim 364,1965$

5) Schneider, C. H. \& A. L. De Weck: A new chemical aspect of penicillin allergy: The direct reaction of penicillin with $\varepsilon$-amino groups. Nature 208:57 59, 1965

6) De WeCK, A. L. \& G. BLum: Recent clinical and immunological aspects of penicillin allergy. Intern. Arch. Allergy Appl. Immunol. $27: 221 \sim 256,1965$

7) Wagner, E. S.; W. W. Davis \& M. Gorman: The reaction of benzylpenicillenic acid with thiol-containing compounds. The formation of a possible penicillin antigenic determinant. J. Med. Chem. $12: 483 \sim 487,1969$

8) Nakken, K. F.; L. Eldjarn \& A. Pihl: The mechanism of inactivation of penicillin by cysteine and other mercaptoamines. Biochem. Pharmacol. $3: 89 \sim 100,1960$. 